\title{
SARCOMA SINOVIAL PRIMARIO PULMONAR: UN CASO INUSUAL EN PEDIATRÍA
}

Dr. Jürg Niederbacher V. ' , Dr. Carlos E. Garavito B. ${ }^{2}$, Dr. Diego Estupiñán P. ${ }^{3}$, Dra. Isabel C. Bolívar A. ${ }^{4}$ Adriana P. Rocha R. ${ }^{5}$ y Ginna P. Lozano C. ${ }^{5}$

1.- Pediatra neumólogo, profesor titular Universidad Industrial de Santander.

2.- Cirujano de tórax, profesor Universidad Industrial de Santander.

3.- Pediatra hemato-oncólogo, profesor de cátedra Universidad Industrial de Santander.

4.- Patóloga.

5.- Estudiantes Medicina, Universidad Industrial de Santander. Bucaramanga, Santander, Colombia.

\section{INTRODUCCIÓN}

El sarcoma sinovial primario de pulmón (SSPP) es un tumor raro que constituye menos del $0,1 \%$ de las neoplasias pulmonares primarias. Principalmente se presentan en adultos jóvenes con una localización habitual en parénquima o pleura. Se informa una presentación extremadamente rara de SSPP, manifestado como una masa endobronquial, representando el primer caso publicado en la literatura en un paciente pediátrico, siendo por ello conveniente su socialización. Este es nuestro objetivo con la publicación del caso clínico.

\section{CASO CLÍNICO}

Paciente masculino de siete años, con antecedente de aparentes neumonías recurrentes, tres en siete meses de evolución; en la última consulta con médico pediatra, refirió que presentaba desde hacía dos días sibilancias, dificultad respiratoria y fiebre. Ante la sospecha de neumonía, se realizó radiografía de tórax, que mostró opacidad del pulmón derecho interpretada como neumonía, reactantes de fase aguda inespecíficos, no se realizaron hemocultivos y se administró tratamiento antibiótico intrahospitalario durante 10 días. En cita de control una semana después, presentaba buena condición clínica, pero persistía compromiso "neumónico" en lóbulo inferior derecho (LID) y lóbulo medio (LM), por lo que es referido a neumología pediátrica. Aquí se indagó sobre los antecedentes previos a la enfermedad actual, los que no eran relevantes, con esquema de vacunación completo para la edad. En el examen físico se encontró condición nutricional normal, disminución del murmullo vesicular y de los ruidos respiratorios en el pulmón derecho, sin otros hallazgos relevantes. Se revisó secuencia radiográfica y se evidenció que presentaba desde hacía 9 meses un componente atelectásico recurrente en LID y LM, notándose mejoría de atelectasias entre uno y otro episodio de las aparentes neumonías (Figura 1, A-B-C).

\section{RESUMEN}

El sarcoma sinovial primario de pulmón (SSPP) localizado en bronquio, es una entidad no reportada en la edad pediátrica. Se presenta el caso de un niño de siete años con antecedente de aparentes neumonías recurrentes derechas de siete meses de evolución; en la evaluación por neumología pediátrica se destaca en las radiografías de tórax, la presencia de atelectasias recurrentes en lóbulo medio e inferior derecho, por lo que se realiza broncoscopia, donde se observa una masa obstruyendo el $100 \%$ de la luz del bronquio fuente derecho y se sospecha tumor carcinoide. Se realiza extirpación de masa endobronquial con fines diagnósticos y terapéuticos, incluyendo resección segmentaria bronquial y anastomosis término-terminal. El respectivo análisis inmunohistoquímico muestra hallazgos sugestivos de sarcoma sinovial monofásico. Se descarta compromiso tumoral extrapulmonar, por lo que se diagnostica como tumor primario de bronquio. Se administraron 7 ciclos de quimioterapia y 31 sesiones de radioterapia. Actualmente en control, sin evidencia de metástasis, tumores residuales o recidivas.

Palabras claves: Sarcoma sinovial, neoplasia pulmonar, neoplasia bronquial primaria en pediatría, atelectasia.

\section{ABSTRACT}

Primary Synovial Sarcoma of Lung (PSSL) located in the bronchus is an unreported entity in pediatric age. We present the case of a 7-year-old child with a history of apparent recurrent right pneumonia of 7 months of evolution; in the evaluation by pediatric pulmonology, the presence of recurrent atelectasis in the middle and lower right lobe is highlighted on chest $X$-rays, so bronchoscopy is performed, where a mass is observed obstructing $100 \%$ of the right bronchus lumen and carcinoid tumor is suspected. Endobronchial mass resection is performed for diagnostic and therapeutic purposes, including bronchial segmental resection and terminoterminal anastomosis. The respective immunohistochemical analysis shows suggestive findings of monophasic synovial sarcoma. Extrapulmonary tumor involvement is ruled out, so it is diagnosed as a primary bronchial tumor. 7 cycles of chemotherapy and 31 sessions of radiation therapy are given. Currently in control, with no evidence of metastasis, residual tumors, or recurrence.

Keywords: Synovial sarcoma, Lung Neoplasms, Pediatric Primary Bronchial Neoplasm, Atelectasis.

Con diagnóstico de atelectasia recurrente, se evalúa con broncoscopia flexible, encontrándose una masa redonda en bronquio fuente derecho (BFD) proximal, que ocluía el 100\% de luz bronquial, lisa, brillante, vascularizada, que no pudo ser sorteada con el fibrobroncoscopio (Figura 2A). Ante sospecha de tumor carcinoide bronquial se decide no practicar biopsia durante el proceso endoscópico por las potenciales complicaciones. Concomitantemente se había solicitado TAC de tórax que ubicó y delimitó la masa proximal en (BFD), correlacionándose con la imagen endoscópica (Figura 2B).

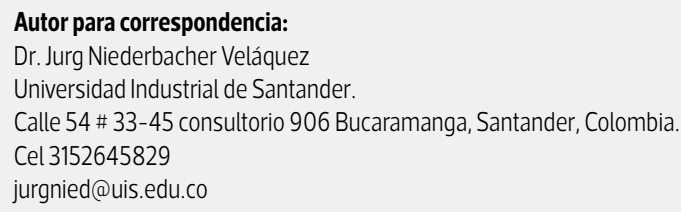



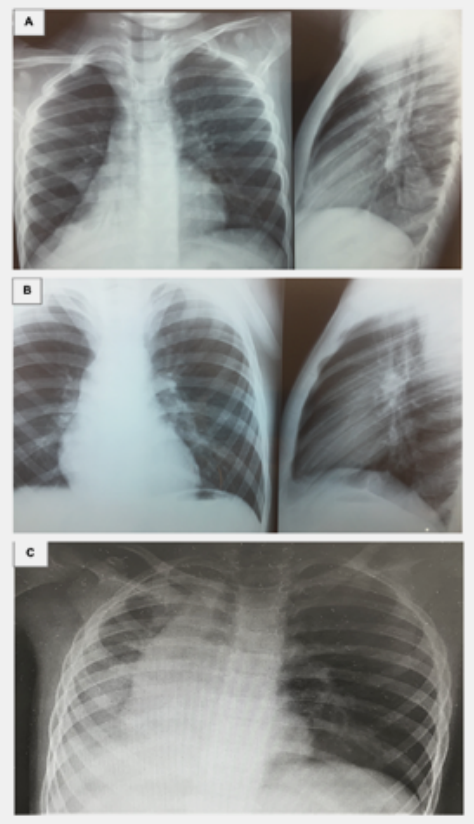

Figura 1. (A) Radiografía de tórax de agosto 11 de 2017: atelectasia de lóbulos inferior y medio del pulmón derecho; (B) Radiografía de tórax de noviembre 14 de 2017: mejoría de atelectasia; (C) Radiografía de tórax de marzo 2018: recurrencia de atelectasia del pulmón derecho.

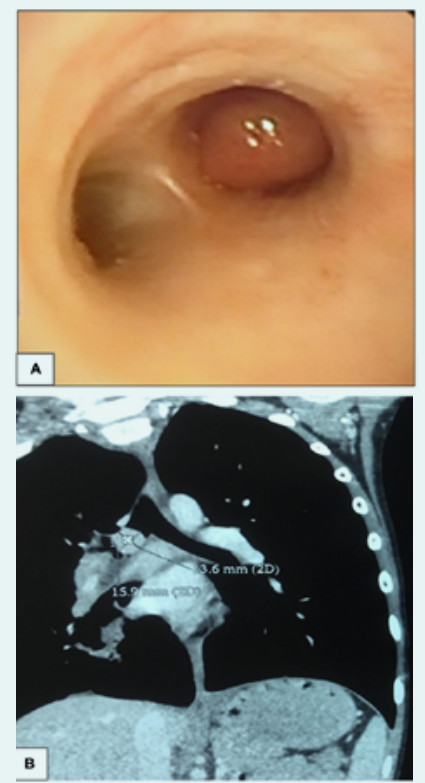

Figura 2. (A) Broncoscopia flexible en abril 2 de 2018: masa bronquio fuente derecho proximal, redonda, lisa, brillante, vascularizada, ocluyendo $100 \%$ de su luz; (B) Tomografía axial computadorizada del tórax: masa con densidad de tejidos blandos que ocluye bronquio fuente derecho proximalmente.
Intraoperatoriamente se encontró una lesión infiltrativa de aproximadamente $2 \mathrm{~cm}$ de diámetro, localizada externamente en pared membranosa de BFD e intermediario. Se extirpó la masa endobronquial con fines diagnósticos y terapéuticos, incluyendo resección bronquial en manguito del segmento comprometido con anastomosis término terminal.

El informe de patología reportó lesión tumoral hipercelular de células fusiformes de escaso citoplasma y núcleos elongados hipercromáticos atípicos, que se mezclaban con vasos sanguíneos de paredes delgadas con extravasación de glóbulos rojos. La actividad mitótica tuvo hasta 4 mitosis en 10 campos (Figura 3-A). Los estudios de inmunohistoquímica fueron positivos para células tumorales con CD99, actina de músculo liso y positividad focal con citoqueratina cóctel AE1- AE3 (Figura 3-B), antígeno epitelial de membrana y $\mathrm{BCl}-2$ y Fli-1. Fueron negativas con proteína S100, CD57, desmina, miogenina, h-caldesmon, actina de músculo específico, CD34, CD31, HHV-8, cromogranina y colágeno IV. El índice de proliferación celular fue variable entre 20 y $30 \%$. Se determinó que los hallazgos eran compatibles con lesión sarcomatoide indiferenciada, que sugería en primer lugar sarcoma sinovial monofásico. No se realizaron estudios de biología molecular. La evaluación clínica e imagenoló-

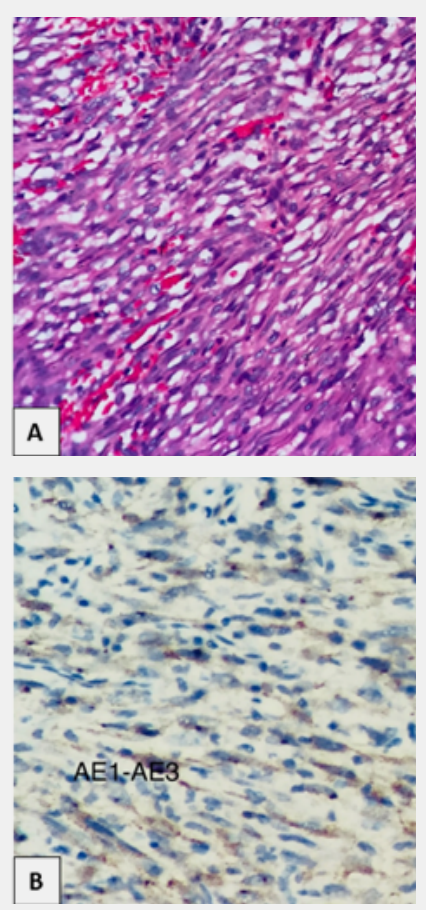

Figura 3. (A) Estudio histológico de lesión tumoral. (B) Estudio de inmunohistoquímica, positividad focal con citoqueratina cóctel AE1- AE3. gica exhaustiva con PET CT excluyó cualquier tumor primario extratorácico, confirmando el diagnóstico de SSPP en este caso.

Se completaron 7 ciclos de quimioterapia, según protocolo ARST 0332 brazo C, y radioterapia en 31 fracciones con fotones de alta energía con una dosis total de 5580 cgy. Esta última se consideró para control local, ya que un borde fue positivo en la patología. No se pudo realizar el estudio del gen de fusión SS18-SSX para confirmar el diagnóstico desde el punto de vista genético. A tres años del diagnóstico inicial, el paciente muestra evolución favorable, sin aparentes metástasis, ni tumores residuales, además con evolución radiográfica normal (figura 4). La exposición de este caso fue aprobada por el comité de bioética institucional.

\section{DISCUSIÓN}

Aunque se pensaba que el sarcoma sinovial (SS) surgía en asociación con la membrana sinovial, ahora se conoce que este no es el caso, y que estos tumores pueden aparecer en cualquier ubicación anatómica. El SS representa aproximadamente el $5-10 \%$ de los sarcomas de tejidos blandos, y de éstos, más del $80 \%$ surgen alrededor de las rodillas; sin embargo, se han descrito en diferentes localizaciones anatómicas (1). El origen primario en pulmón
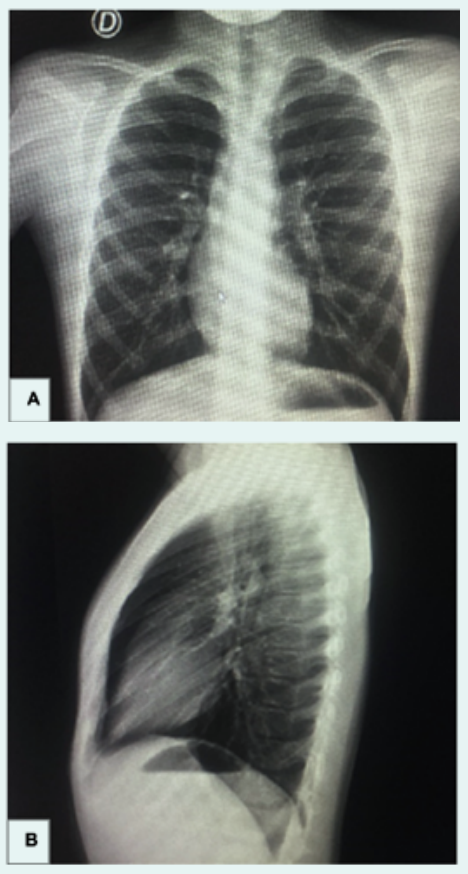

Figura 4 A y B. Radiografía de tórax AP y lateral de enero 23 de 2021 (tres años luego del tratamiento médico-quirúrgico): Normal. 
es inusual, con mayor frecuencia de compromiso metastásico en esta localización. El SSPP constituye el $0,1 \%$ de los tumores primarios pulmonares (2), y suele afectar a adultos jóvenes, con una edad media de 31 a 50 años, sin predilección por algún sexo (1).

El sarcoma sinovial intratorácico surge más comúnmente del pulmón adyacente a la pleura y el mediastino. El SSPP afecta a ambos sexos por igual sin preferencia por ningún hemitórax. La morfología, las propiedades de inmunotinción, las características citogenéticas y la estrategia de manejo de SSPP son similares a las de la SS de tejidos blandos. Histológicamente, hay dos tipos principales de SS: monofásico y bifásico, con una característica de diferenciación deficiente que se observa en ambos tipos. La mayoría de los pacientes se presentan con grandes masas intratorácicas con derrame pleural ipsilateral o sin él. La invasión ósea o adenopatía mediastínica es muy rara. El SS se caracteriza por una translocación cromosómica específica que produce el gen de fusión SS18-SSX en más del $90 \%$ de los casos. La identificación de este gen de fusión sigue siendo el estándar de oro para el diagnóstico en presencia de una histología e inmunofenotipo consistentes (1)

Las manifestaciones clínicas dependen de la localización del SSPP, entre estas las más frecuentes son tos, hemoptisis, disnea y dolor torácico; sin embargo, es frecuente encontrar pacientes asintomáticos con hallazgos incidentales (1). En nuestro paciente se consideró como diagnóstico principal, un tumor carcinoide endobronquial, puesto que es la neoplasia pulmonar maligna primaria más común en niños (1); además, las manifestaciones clínicas que presentó, aparente neumonía recurrente y atelectasias, son hallazgos frecuentes en esta patología (2). Es de notar que las atelectasias recurrentes o persistentes por compromiso endoluminal abarcan una serie de condiciones como cuerpos extraños, tapones de moco y tumores endobronquiales entre otras $(3,4,5)$.

La localización del SSPP es pleural, parenquimatosa o endobronquial; esta última de presentación extremadamente rara, con solo nueve casos descritos en la literatura, en pacientes entre los 34 y 73 años. (Ver tabla 1) (6-14).

Macroscópicamente, varían en tamaño desde menos de un $\mathrm{cm}$ hasta más de $20 \mathrm{~cm}$ al momento del diagnóstico, son masas bien delimitadas, pero sin encapsular, blandas, de color canela con focos de necrosis, hemorragia y cambios quísticos (15). Microscópicamente, la mayoría de los SSPP son de tipo monofásico, los cuales constan de un componente de células fusiformes fasciculares, con cantidades

Tabla 2. Estudios sobre efectos respiratorios de la exposición a material particulado aéreo proveniente de incendios forestales.

\begin{tabular}{|c|c|c|c|c|c|}
\hline Estudio & Edad y Sexo & $\begin{array}{l}\text { Presentación } \\
\text { clínica }\end{array}$ & $\begin{array}{c}\text { Caracteristicas de } \\
\text { tumor }\end{array}$ & Tratamiento & Resultado \\
\hline $\begin{array}{l}\text { Niwa y } \\
\text { col. (6) }\end{array}$ & $\begin{array}{l}\text { Mujer de } 42 \\
\text { años }\end{array}$ & Hemoptisis & $\begin{array}{l}\text { Tumor polipoide del } \\
\text { BFI de } 2,5 \mathrm{~cm}\end{array}$ & $\begin{array}{l}\text { Lobectomía y } \\
\text { quimioterapia } \\
\text { adyuvante }\end{array}$ & $\begin{array}{l}\text { Recurrencia } \\
\text { después de } \\
\text { tres años }\end{array}$ \\
\hline $\begin{array}{l}\text { Essary y } \\
\text { col. (7) }\end{array}$ & $\begin{array}{l}\text { Hombre de } \\
69 \text { años }\end{array}$ & $\begin{array}{l}\text { Disnea, tos, } \\
\text { hemoptisis por } \\
\text { cinco meses }\end{array}$ & $\begin{array}{l}\text { Tumor de } 3,5 \mathrm{~cm} \\
\text { en LSD, que se } \\
\text { extiende hacia los } \\
\text { bronquios }\end{array}$ & Lobectomía & $\begin{array}{l}\text { Recurrencia a } \\
\text { los dos años, } \\
\text { falleció a los } \\
\text { dos años } \\
\text { de causa no } \\
\text { relacionada }\end{array}$ \\
\hline $\begin{array}{l}\text { Watanabe } \\
\text { y col. (8) }\end{array}$ & $\begin{array}{l}\text { Hombre de } \\
34 \text { años }\end{array}$ & Tos y hemoptisis & $\begin{array}{l}\text { Tumor en LII con } \\
\text { masa polipoide } \\
\text { endobronquial }\end{array}$ & Lobectomía & $\begin{array}{l}\text { Libre de } \\
\text { enfermedad }\end{array}$ \\
\hline $\begin{array}{l}\text { Kumar y } \\
\text { col.(9) }\end{array}$ & $\begin{array}{l}\text { Mujer de } 35 \\
\text { años }\end{array}$ & Tos y disnea & $\begin{array}{l}\text { Tumor en LSI de seis } \\
\text { cm con extensión } \\
\text { endobronquial }\end{array}$ & $\begin{array}{l}\text { Neumonecto- } \\
\text { mía y quimiote- } \\
\text { rapia basada en } \\
\text { adriamicina }\end{array}$ & $\begin{array}{l}\text { Libre de en- } \\
\text { fermedad un } \\
\text { año después } \\
\text { de la cirugía }\end{array}$ \\
\hline $\begin{array}{l}\text { Li Jing y } \\
\text { col. (10) }\end{array}$ & $\begin{array}{l}\text { Mujer de } 36 \\
\text { años }\end{array}$ & Tos y hemoptisis & $\begin{array}{l}\text { Tumor endobron- } \\
\text { quial en el BFI que } \\
\text { causa colapso } \\
\text { completo de lóbulo } \\
\text { inferior izquierdo }\end{array}$ & $\begin{array}{l}\text { Neumonec- } \\
\text { tomía }\end{array}$ & No reportado \\
\hline $\begin{array}{l}\text { Tandon y } \\
\text { col. (11) }\end{array}$ & $\begin{array}{l}\text { Mujer de } 45 \\
\text { años }\end{array}$ & $\begin{array}{l}\text { Tos y hemoptisis } \\
\text { por seis meses }\end{array}$ & $\begin{array}{l}\text { Masa en LID de } 2,5 \times \\
2 \mathrm{~cm} \text { con extensión } \\
\text { endobronquial }\end{array}$ & Lobectomía & $\begin{array}{l}\text { Pérdida de } \\
\text { seguimiento }\end{array}$ \\
\hline $\begin{array}{l}\text { Al - Ani y } \\
\text { col. (12) }\end{array}$ & $\begin{array}{l}\text { Mujer de } 68 \\
\text { años }\end{array}$ & Tos por tres meses & $\begin{array}{c}\text { Masa en LSD de } \\
\text { tejido blando de } \\
5,6 \times 5,4 \times 3 \mathrm{~cm} \\
\text { con componente } \\
\text { endobronquial }\end{array}$ & Lobectomía & $\begin{array}{l}\text { Libre de } \\
\text { enfermedad, } \\
\text { seguido en } \\
\text { exploraciones } \\
\text { seriadas }\end{array}$ \\
\hline $\begin{array}{l}\text { Leonard y } \\
\text { col. (13) }\end{array}$ & $\begin{array}{l}\text { Hombre de } \\
73 \text { años }\end{array}$ & $\begin{array}{l}\text { Tos productiva de } \\
\text { esputo amarillo, } \\
\text { disnea }\end{array}$ & $\begin{array}{l}\text { Nódulo pulmonar } \\
\text { en el LII de } 2,0 \times 2,4 \\
\text { cm con extensión } \\
\text { endobronquial }\end{array}$ & Lobectomía & $\begin{array}{c}\text { Libre de } \\
\text { enfermedad } \\
\text { cinco meses } \\
\text { después de la } \\
\text { cirugía }\end{array}$ \\
\hline $\begin{array}{l}\text { Katragadda } \\
\text { y col. (14) }\end{array}$ & $\begin{array}{l}\text { Hemoptisis } \\
\text { Hombre de } \\
62 \text { años }\end{array}$ & $\begin{array}{l}\text { Sin síntomas, ha- } \\
\text { llazgo incidental }\end{array}$ & $\begin{array}{c}\text { Masa de } 5,7 \times 6,3 \\
\times 5,9 \mathrm{~cm} \text { en el LSD } \\
\text { con extensión endo- } \\
\text { bronquial }\end{array}$ & $\begin{array}{c}\text { Quimioterapia } \\
\text { y radioterapia } \\
\text { prequirúrgica } \\
\text { debido al tama- } \\
\text { ño de la masa }\end{array}$ & No reportado \\
\hline
\end{tabular}

LSD: lóbulo superior derecho, LID: lóbulo inferior derecho, LSI: lóbulo superior izquierdo, LII: lóbulo inferior izquierdo, BFI: bronquio fuente izquierdo.

moderadas de citoplasma eosinofílico pálido, núcleos redondos a alargados y ahusados, cromatina vesicular y nucleolos pequeños y distintos, en ausencia de componente epitelial, que lo diferencia de los sarcomas bifásicos (16)

El componente glandular del sarcoma sinovial expresa citoqueratinas, incluidas AE1 / AE3. Se evidencia además una fuerte positividad focal para el antígeno de la membrana epitelial (EMA) y la queratina y, por otro lado, la vimentina muestra una fuerte positividad difusa. Otras inmunotinciones, incluida la desmina, la actina del músculo liso y la proteína S-100, son negativas (17). También se ha observado positividad para la proteína Bcl-2, en aproximadamente el $79 \%$ de los casos, en el componen- te de células fusiformes del sarcoma sinovial, pero, la especificidad de este marcador es baja y la reactividad de BCL2 está presente en una variedad de otros tumores de tejidos blandos $(16,17)$

Estudios demuestran que un cambio genético, la translocación t $(X ; 18)$ (p11.2; q11.2), se identifica en el $90 \%$ de los casos; en los casos restantes, el diagnóstico se debe basar en las características inmunohistoquímicas e inmunofenotípicas (1)

No existen pautas de tratamiento debido a la rareza de la presentación endobronquial, sin embargo, todos los pacientes adultos de los casos descritos requirieron cirugía, en su mayoría lobectomía y el resto neumectomía, 
recibiendo tres pacientes tratamiento adicional con quimioterapia o radiación.

El tratamiento multimodal que incluye escisión amplia, quimioterapia y radioterapia es el pilar de la terapia. El SS generalmente se considera un tumor relativamente quimiosensible y el tratamiento con adriamicina sola 0 en combinación con ifosfamida sigue siendo la quimioterapia estándar en la enfermedad metastásica. Los ensayos controlados aleatorios y los estudios observacionales han demostrado que el régimen basado en ifosfamida mejora la supervivencia en los sarcomas de tejidos blandos de las extremidades. La combinación de ifosfamida y adriamicina se puede utilizar para el alivio rápido de los síntomas y en pacientes que se planifica la resección curativa de metástasis. La radioterapia sola o combinada con cirugía se usa para lograr un excelente control local de la enfermedad. La tasa de respuesta global es de alrededor del 50\% con regímenes que contienen ifosfamida y doxorrubicina (1).

En cuanto a la evolución y el pronóstico, se ha descrito que en población menor a 21 años, aproximadamente el $40 \%$ de los pacientes con SS sobreviven más de cinco años desde la recurrencia. Adicionalmente, se ha relacionado el tamaño inicial del tumor (cinco centímetros o más) a una primera recurrencia con metástasis a distancia, lo que podría indicar una biología más agresiva, y por consiguiente un peor pronóstico (18). En cuanto al SSPP, en el estudio de cohorte de Lan y colaboradores del 2015, en pacientes entre los 12 a 60 años, la mediana del tiempo de supervivencia fue de 14,5 meses, con supervivencia específica de la enfermedad a los 2 años de 27,7\%. Además, describieron una asociación significativa en la mejoría de la supervivencia con la resección quirúrgica y la ausencia de tumor residual. En el caso de la presentación endobronquial, solo dos pacientes tuvieron recurrencia de la enfermedad años después de su resección (19).

Generalmente, los SS se consideran tumores de alto grado con mal pronóstico. El SS tiene una tendencia a la recurrencia tardía y la metástasis; por lo tanto, se recomienda un seguimiento más prolongado de más de 10 años. Los pacientes con enfermedad localizada tienen una buena probabilidad de curación con cirugía y radioterapia, y los márgenes negativos y la radioterapia adyuvante son factores cruciales para determinar la supervivencia local libre de recidiva.

Las nuevas terapias dirigidas al oncogén de fusión, la vacuna peptídica derivada de SS18-SSX, el receptor del factor de crecimiento epidérmico y el factor de crecimiento endotelial vascular son la esperanza futura en el manejo del SS (1).

\section{CONCLUSIONES}

El SSPP, es un tumor muy raro en pediatría con pronóstico incierto; en este caso el diagnóstico temprano y manejo oportuno con cirugía y quimio-radioterapia ha favorecido su evolución y pronóstico.

LoS autores declaran no presentar conflicto de interés.

\section{AGRADECIMIENTOS}

Instituto Neumológico del Oriente (Bucaramanga, Santander, Colombia), en donde se diagnosticó el caso y al paciente y familia que autorizaron su publicación.

\section{Revisores de este artículo}

Dra. Lilian Rubilar

Pediatra Broncopulmonar

Hospital Dr. Exequiel González Cortés.

Santiago, Chile.

\section{Dra. Ilse Contreras}

Pediatra Broncopulmonar

Profesora Asociada de la Facultad de Medicina. Universidad del Desarrollo

Santiago, Chile

Fecha de recepción: abril 2021

Fecha de publicación: diciembre 2021
REFERÉNCIAS

1. Panigrahi M, Pradhan G, Sahoo N, Mishra P, Patra S, Mohapatra P. Primary pulmonary synovial sarcoma: A reappraisal. J Cancer Res Ther [Internet]. 2018 [Cited 2021 Sept 03]; 14(3):481-9. Available from: https://pubmed. ncbi.nlm.nih.gov/29893303/

2. Hartel P, Fanburg-Smith J, Frazier A, Galvin J, Lichy J, Shilo K, et al. Primary pulmonary and mediastinal synovial sarcoma: a clinicopathologic study of 60 cases and comparison with five prior series. Mod Pathol [Internet]. 2007 [Cited 2021 May 01]; 20(7):760-69. Available from: https://www.nature.com/articles/3800795

3. Peroni DG, Boner AL. Atelectasis: mechanisms, diagnosis and management. Paediatr Respir Rev [Internet]. 2000 [Cited 2021 May 01]; 1(3):274-8. Available from: https:// pubmed.ncbi.nlm.nih.gov/12531090/

4. Johnston C, Brunow W, Atelectasis: mechanisms, diagnosis and treatment in the pediatric patient. Rev Assoc Med Bras [Internet]. 2008 [Cited 2021 May 01]; 54(5): 455-60. Available from: https://www.scielo.br/j/ram$\mathrm{b} / \mathrm{a} / \mathrm{tm} X \times$ SVnx5MHrnTsQYVswkKq/?lang=pt

5. Talamoni H, Pisapia N, Buendía J. Broncoscopía flexible en niños con atelectasias persistentes. Serie de casos. Arch Argent Pediatr [Internet]. 2015 [Cited 2021 May 01]; 113(2):e106-8. Available from: https://www.sap.org.ar/ docs/publicaciones/archivosarg/2015/v113n2a17.pdf

6. Niwa H, Masuda S, Kobayashi C, Oda Y. Pulmonary synovial sarcoma with polypoid endobronchial growth: A case report, immunohistochemical and cytogenetic study. Pathol Int [Internet]. 2004 [Cited 2021 May 01]; 54(8):611-5. Available from: https://onlinelibrary.wiley.com/doi/ abs/10.1111/j.1440-1827.2004.01670.x

7. Essary LR, Vargas SO, Fletcher CDM. Primary pleuropulmonary synovial sarcoma. Cancer [Internet]. 2002 [Cited 2021 May 01]; 94(2):459-69. Available from: https:// acsjournals.onlinelibrary.wiley.com/doi/abs/10.1002/ cncr.10188

8. Watanabe R, Kamiyoshihara M, Kaira K, Motegi A, Takise A. Spontaneous Expectoration of Primary Pulmonary Synovial Sarcoma. J Thorac Oncol [Internet]. 2006 [Cited 2021 May 01]; 1(9):1025-6. Available from: https://www. jto.org/article/S1556-0864(15)31638-5/fulltext

9. Kumar R, Menon S, Desai S, Pramesh C, Menon H, Jambhekar N. Primary endobronchial synovial sarcoma confirmed by SYT-SSX1 fusion gene transcript by reverse transcriptase polymerase chain reaction. Indian J Pathol Microbiol [Internet]. 2009 [Cited 2021 May 01]; 52(4):520-3. Available from: https://www.ijpmonline. org/article.asp?issn=0377-4929

10. Li JJ, Yang T, Ismail K. Endobronchial synovial sarcoma: a rare tumour in an unusual location. Pathology [Internet]. 2013 [Cited 2021 May 01]; 45:S75. Available from: http://www.sciencedirect.com/science/article/pii/ S0031302516317834

11. Tandon A, Uppin S, Patnaik S, Sundaram C. Primary endobronchial synovial sarcoma. Astrocyte [Internet]. 2014 [Cited 2021 May 01]; 1(2):162-5. Available from: http:// www.astrocyte.in/article.asp?issn=2349-0977

12. Al-Ani Z, Naqvi J, Oh TC. Primary pleuropulmonary synovial sarcoma mimicking a carcinoid tumor: Case report and literature review. Radiol case reports [Internet]. 2016 [Cited 2021 May 01]; 11(2):45-9. Available from: https:// pubmed.ncbi.nlm.nih.gov/27257447

13. Leonard R, Schultz C, Hadique S. An unusual endobronchial lesion: expanding the differential diagnosis. Respirol case reports [Internet]. 2019 [Cited 2021 May 01]; 7(5):e0429. Available from: https://pubmed.ncbi.nlm. nih.gov/31019717

14. Katragadda R, Gidda K, Dogra R, Katragadda S. Primary Pulmonary Synovial Sarcoma: Endobronchial Mass in 
a Non-Smoker with Asbestos Exposure. Am J Respir Crit Care Med 2 [Internet]. 2020 [Cited 2021 May 01]; 201:A5870. Available from:https://www.atsjournals. org/doi/10.1164/ajrccm-conference.2020.201.1_MeetingAbstracts.A5870

15. Sholl LM. Chapter 5 Tumors of the Lungs and Pleura. Diagnostic Histopathology of Tumors, Fifth Edition. Elsevier Inc. [Internet]. 2021 [Cited 2021 May 01]; 225-72. Available from: https://www.clinicalkey.es/\#!//content/3-s2.0-B9780323428606000065

16. Doyle LA, Hornick JL. Chapter 4 Immunohistology of Neoplasms of Soft Tissue and Bone. Diagnostic Immunohistochemistry, Fifth Edition. Elsevier Inc. [Internet]. 2019 [Cited 2021 May 01]. 82-136. Available from: https://www.clinicalkey.es/\#!//content/3-s2.0-B9780323477321000040

17. Pan M, Merchant M. Risk Factors Including Age, Stage and Anatomic Location that Impact the Outcomes of Patients with Synovial Sarcoma. Med Sci [Internet]. 2018 [Cited 2021 Sept 031; 6(1):21-34. Available from: https://www. mdpi.com/2076-3271/6/1/21

18. Scheer M, Dantonello T, Hallmen E, Blank B, Sparber-Sauer M, Vokuhl C, et al. (2016). Synovial Sarcoma Recurrence in Children and Young Adults. Ann Surg Oncol [Internet]. 2016 [Cited 2021 Sept 03]; 23: 618-26. Available from: https://link.springer.com/article/10.1245\%2Fs10434-016-5535-2

19. Lan T, Chen H, Xiong B, Zhou T, Peng R, Chen M, et al. Primary pleuropulmonary and mediastinal synovial sarcoma: a clinicopathologic and molecular study of 26 genetically confirmed cases in the largest institution of southwest China. Diagn Pathol [Internet]. 2016 [Cited 2021 Sept 03]; 11(1):62-76. Available from: https://diagnosticpathology.biomedcentral.com/articles/10.1186/ s13000-016-0513-3 